
\title{
23 Research Square \\ Feasibility Study of Transfacet Sacroiliac Screw Fixation Based on Mimics
}

\author{
Yan-ping Din \\ Soochow University \\ Qu-dong Yin \\ Soochow University \\ Shuai Liu \\ Soochow University \\ Dong Li \\ Nantong University \\ Yong-wei Wu \\ Soochow University \\ Yan Jiang ( $\sim$ 13665150065@163.com )
}

\section{Research article}

Keywords: Sacroiliac joint, Sacroiliac screw, Transfacet, Computed tomography, Mimics, Anatomy

Posted Date: July 30th, 2020

DOI: https://doi.org/10.21203/rs.3.rs-42564/v1

License: (c) (i) This work is licensed under a Creative Commons Attribution 4.0 International License.

Read Full License 


\section{Abstract}

Background: Sacroiliac screw (SIS) has become an effective internal fixation method for sacral fracture and sacroiliac joint dislocation. However, classic placement of SIS has some defects such as possibility of nerve injury with a learning curve and contraindications. An alternative to the classic placement of SIS is expected.

Objective: To explore the feasibility of transfacet sacroiliac screw (TFSIS), so as to provide an alternative to the classic placement of SIS.

Methods $\llbracket C T$ scan data of pelvis in 60 healthy adults including 30 males and 30 females with an average age of 45 years (range 20-70 years), were transferred into a PC. The anatomical parameters of screw channel of TFSIS were measured by simulating the placement of TFSIS by Mimics 16.0 software on the PC. Secondly, according to the anatomical parameters of each pelvis, $5.0 \mathrm{~mm}$ and $6.0 \mathrm{~mm}$ screws were used respectively to simulate the placement of TFSIS in 30 pelvises to observe the effect of placement.

Results $\triangle$ The length of screw channel was $(10.84 \pm 0.93) \mathrm{cm}$, the distance between the insertion point and the center of the superior facet of $\mathrm{S} 1$ was $(1.14 \pm 0.93) \mathrm{mm}$, the distance between the exit point and the upper -posterior border of acetabulum was $(5.73 \pm 2.57) \mathrm{mm}$, the anteversion angle between the central axis of the screw channel and the line parallel to the upper endplate of $S 1$ was $(53.96 \pm 3.94)^{\circ}$, the outward angle between the central axial of the screw channel and the longitudinal axis of the trunk was $(47.47 \pm 5.13)^{\circ}$, the safety angle in sagittal plane was $(13.91 \pm 2.92)^{\circ}$, the safety angle in coronal plane was $(8.57 \pm 1.63)^{\circ}$, the height was $(11.91 \pm 1.47) \mathrm{mm}$, and the width was $(7.75 \pm 0.89) \mathrm{mm}$. Within the channel for $5.0 \mathrm{~mm}$ and $6.0 \mathrm{~mm}$ screws accounted for $100 \%$.

Conclusions $\rrbracket$ Placement of TFSIS with a diameter of 5.0-6.0 $\mathrm{mm}$ and a length of $90 \mathrm{~mm}$ is safe and feasible, which may be used as an alternative to the classic placement of SIS.

\section{Highlights}

The transfacet sacroiliac screw (TFSIS) is different from the classic sacroiliac screw, which inserts from the posterior of S1, forward, downward and outward, passes through the pedicle and lateral mass of S1, as well as sacroiliac joint, and finally enters the ilium. This study showed that placement of TFSIS is safe and feasible.

\section{Background}

Sacroiliac screw (SIS) has become an effective internal fixation method for sacral fracture and sacroiliac joint dislocation [1,2]. The classic SIS internal fixation is placed percutaneously, which passes from the posterolateral side of the ilium through the sacroiliac joint into the vertebral body of the sacrum $[3,4]$. The anterior part of the screw channel is close to the iliac vascular and nerve, and the upper and lower parts of the channel are close to the lumbar and sacral nerves. In order to reduce the complications of nerve 
injury, placement of SIS requires repeated fluoroscopy at the pelvic outlet, inlet and lateral views to confirm the correct placement position, with more radiation exposure and a risk of vascular and nerve injury. The incidence of screw malposition and nerve injury was $2 \%-68 \%$ and $0.5 \%-7.9 \%$, respectively $[5,6,7]$. Recently, a variety of computer-assisted techniques and robot-assisted navigation have been developed that require detailed anatomical knowledge and extensive surgical experience, with a learning curve $[5,6,8]$. In addition, classic placement of SIS has contraindications, such as in patients with severe osteoporosis and local fractures or infection at the insertion position of the ilium. Therefore, an alternative to the classic placement of SIS is expected.

After studying the pelvic anatomy and radiographs, we found a novel sacroiliac joint screw channeltransfacet sacroiliac screw (TFSIS), which is different from the classic SIS. It inserts from the back of S1, forward, downward and outward, passes through the pedicle and lateral mass of S1, as well as sacroiliac joint, and finally enters the ilium. This placement of SIS can avoid injury of vessels and nerves with the characteristics of safe placement and indication for patients with severe osteoporosis. To our knowledge, there is no report of TFSIS. The purpose of this study was to investigate the feasibility of TFSIS placement, so as to provide a basis for clinical application.

\section{Methods}

\subsection{Observation on the range of placement of TFSIS}

The insertion point was located at the center of the facet, which is between L5 and S1 nerve roots. The exit point was located near the superior- posterior border of the acetabulum. The medial wall of the screw channel is the arcuate line, the lateral wall of the screw channel is the posterolateral wall of the spinal canal of S1 and the outer layer of the ilium. The inferior wall of the screw channel is the line between the sacral foramen of S1 and the ischial notch. The upper wall of the screw channel is the sacral wing slope, the surface of the sacroiliac joint and the bottom of the inner wall of the great pelvis.

\subsection{Patients}

The study cohort comprised CT scan data of pelvis in 60 healthy adult patients ( 120 sacroiliac joints). There were 30 males and 30 females, whose age averaged 45 years (range 20 to 70 years); the CT scan data were from the database of our hospital between May, 2019 to April, 2020. In the data selection process, all pelvises found with tumor, fracture or variation were excluded from the research. The study was approved by ethics committee of our hospitals囚LW2020025》.

\subsection{Equipment and scan mode}

A 64-slice CT of GE Optima CT 660 was used to scan the inspected patient lying supinely from head to foot. The scanning range was set from the superior margin of $L 3$ to $5 \mathrm{~cm}$ below the symphysis pubis. The mAs auto-adjust technology was used to obtain the scan parameters: tube voltage $120 \mathrm{kV}$, thickness 1 $\mathrm{mm}$, interval $1.0 \mathrm{~mm}$, pitch 1.172, rotation time $0.75 \mathrm{~s}$, matrix $512 \times 512$, Fov $350 \otimes 400 \mathrm{~mm}$. The raw data 
obtained were reconstructed to $1 \mathrm{~mm}$ thickness, $50 \%$ interval and $300 \mathrm{HU}$ threshold; The CT scan images were stored in DICOM format on CD-ROM. The pelvic scan data in DICOM format were transferred to a $\mathrm{PC}$, and 3D reconstruction of the pelvis was performed by Mimics 16.0 software (Belgium materialise company) on the PC.

\subsection{Measurements}

First, the optimal screw pathway-central axis of the screw channel of TFSIS was determined by simulating the TFSIS fixation. It starts from the back of the spinal canal of S1(insertion point), inserts forward, downward and outward, passes through the pedicle and lateral mass of S1, as well as sacroiliac joint, enters the ilium, and finally penetrates the lateral wall of the ilium (exit point). Then, sagittal and coronal scans were performed on the central axis of the screw channel to measure the anatomical parameters. Secondly, according to the anatomical parameters of each pelvis, $5.0 \mathrm{~mm}$ and $6.0 \mathrm{~mm}$ screws were used respectively to simulate the placement of TFSIS in 30 pelvises to observe the effect of placement. Definitions of the anatomical parameters were as follows (Fig.1):

(1) the insertion point position (M1): the distance from the insertion point of the central axis of the screw channel to the center of the superior facet of S1.

(2) the exit point position (M2): the distance from the exit point of the central axis of the screw channel to the superior- posterior border of the acetabulum.

(3) the length of screw channel $(L)$ : the distance from the insertion point to the exit point of the central axis of the screw channel.

(4) the anteversion angle (E): the angle between the central axis of the screw channel and the line parallel to the upper endplate of $\mathrm{S} 1$ in sagittal plane.

(5) the outward angle (F): the angle between the central axis of the screw channel and the longitudinal axis of the trunk in coronal plane.

(6) the forward safety angle (A): the maximum allowable deviation angle of $\varphi 5 \mathrm{~mm}$ screw within the screw channel in sagittal plane.

(7) the outward safety angle of the screw (B): the maximum allowable deviation angle of $\varphi 5 \mathrm{~mm}$ screw within the screw channel in coronal plane.

(8) the height (D1): the narrowest height of the screw channel in sagittal plane.

(9) the width (D2): the narrowest width of the screw channel in coronal plane.

\subsection{Statistics}


Statistical description and statistical analysis were done with SPSS 13.0. The testing results of left and right sides were compared with matched $t$ testing method. $\mathrm{P}<0.05$ was considered to indicate a statistically significant difference.

\section{Results}

All the data were in line with normal distribution; there was no significant difference between the left side and right side $(P>0.05)$, so the data of both sides were merged. There were no significant differences between male and female $(P>0.05)$. $L$ was $(10.84 \pm 0.93) \mathrm{cm}$, of which, the length on the sacral side was $(5.19 \pm 0.36) \mathrm{mm}$ and $(5.65 \pm 0.35) \mathrm{mm}$ for the iliac side. M1 was $(1.14 \pm 0.93) \mathrm{mm}$. M2 was $(5.73 \pm$ 2.57) $\mathrm{mm}$. E was $(53.96 \pm 3.94)^{\circ}, \mathrm{F}$ was $(47.47 \pm 5.13)^{\circ}$. A was $(13.91 \pm 2.92)^{\circ}, \mathrm{B}$ was $(8.57 \pm 1.63)^{\circ} . \mathrm{M} 1$ was $(11.91 \pm 1.47) \mathrm{mm}$, and $\mathrm{M} 2$ was $(7.75 \pm 0.89) \mathrm{mm}$. The values of parameters were shown in table 1 .

\section{Discussion}

\subsection{Classic SIS fixation}

The existing method of SIS fixation is placed percutaneously under fluoroscopy, the insertion point is located at the posterior- lateral side of the ilium, the insertion direction is inward, forward and upward, and enters the lateral mass and pedicle of the sacrum vertically through the sacroiliac joint surface, and into the vertebrae body. However, the L5 or S1 nerve root may be damaged if the screw deviates too much upward or downward; the iliac vessel and organ may be damaged if the screw deviates too much foreward; the sacral nerve in the spinal canal may also be damaged if the screw deviates too much backward. The screw placement can not be accurately determined by single AP or lateral view $[9,10]$. Therefore, repeated intraoperative fluoroscopic images must be used by examining the inlet and outlet views, especially the standard lateral view with overlap of the anatomic landmark of the iliac cortical density (ICD) for safe insertion of a sacroiliac screw. However, the presence of contrast, intestinal gas, and increased soft tissue density from obesity can cause difficulty in obtaining and interpreting appropriate intraoperative fluoroscopic images. This may lead to malpositioned implants and increased risk of neurovascular injuries. As a result, existing sacroiliac fixation technique has a learning curve and some incidence of screw penetration and nerve injury $[4,5,7,8]$.

\subsection{TFSIS fixation}

The insertion point of TFSIS is located at the center of the facet, which is between the L5 and S1 nerve roots. Because it is placed either under direct vision after a spinal posterior incision or through a minimally invasive method with a aiming device, the position of the insertion point can be controlled; the insertion direction is outward, where is a safe area. Therefore, injury of nerve, iliac vessel and viscera organ can be avoided, i.e. this method is safe.

The medial wall of the screw channel is arcuate line, the lateral wall of the screw channel is the posterolateral wall of the spinal canal of S1 and the outer layer of the ilium. The inferior wall of the screw 
channel is the line between the sacral foramen of S1 and the ischial notch. The upper wall of the screw channel is the sacral wing slope, the surface of the sacroiliac joint and the bottom of the inner wall of the great pelvis. With naked eyes, the range of screw placement is large.

D2 was $(7.75 \pm 0.89)$ smaller than $\mathrm{D} 1(11.91 \pm 1.47) \mathrm{mm} ; \mathrm{A}$ and $\mathrm{B}$ were $(11.91 \pm 1.47)^{\circ}$ and $(8.57 \pm 1.63)^{\circ}$, respectively. Therefore, it is not allowed to use larger diameter screws, we think $\varphi 5.0$ to $6.0 \mathrm{~mm}$ screws are suitable.

$L$ averaged $10.84 \mathrm{~cm}$. However, if the insertion direction deviates inward and forward, the screw channel is longer; if the insertion direction deviates outward and laterally, the screw channel is shorter. Considering that too long screws may penetrate the ilium or the acetabulum, $90 \mathrm{~mm}$ long screw is recommended.

The key to the safe placement of TFSIS is the insertion point position and insertion direction. The insertion point is close to the center of the superior facet of $\mathrm{S} 1(\mathrm{M} 1=1.14 \mathrm{~mm})$. E averaged $53.96^{\circ}, \mathrm{F}$ averaged $47.47^{\circ}$. According to the insertion point and insertion direction of each patient, within screw channel accounted for $100 \%$ for $\varphi 5 \mathrm{~mm}$ and $\varphi 6 \mathrm{~mm}$ screws, which indicated that this method was feasible and safe.

The iliac side of TFSIS fixation is almost all cortical bone, and the screw channel in the iliac side is longer, the anti-pulling force of screw is stronger than that of the classic SIS. Therefore, TFSIS is suitable for patients with severe osteoporosis. The iliac side of classic SIS fixation is about $50 \%$ cancellous bone and $50 \%$ cortical bone, and the stability of screw in patients with severe osteoporosis is poor $[11,12]$.

Therefore, the classic placement of SIS is not indicated in patients with severe osteoporosis and local fractures or infection at the insertion position of ilium. However, these situations are suitable for TFSIS, i.e. TFSIS can be used as an alternative to the classic placement of SIS.

In a word, larger diameter and longer screws should not be used; there were individual differences in the anatomical parameters, so the preoperative 3D CT reconstruction of pelvic should be performed to measure the relevant parameters, individualized screw placement is needed. On the premise of being within the channel, the screw should be placed as close as possible to the medial-anterior of the central axis, so as to maximize the stability and safety effect.

The disadvantages of TFSIS fixation: the diameter of the screw channel in this method is small, only one screw with $\varphi$ 5.0-6.0 $\mathrm{mm}$ can be used. In classic SIS fixation, the width and height of the screw pathway were $(27.7 \pm 1.9) \mathrm{mm}$ and $(20.2 \pm 2.3) \mathrm{mm}$, respectively [4,6], and $1-2 \varphi 6 \mathrm{~mm}$ screws can be placed. Therefore, its anti-bending strength of TFSIS is not as good as that of the classic method.

\section{Conclusions}

Anatomically, TFSIS can be placed into the pelvis to fix sacroiliac joint. The suitable screw diameter is $5.0-6.0 \mathrm{~mm}$ and the length is about $90 \mathrm{~mm}$. The best direction of the screw insertion is $54^{\circ}$ anteversion 
angle and $47^{\circ}$ outward angle. TFSIS placement is not only feasible but also safe. Mimics software helps to accurate individualized placement of TFSIS.

\section{Abbreviations}

SIS. Sacroiliac screw.

TFSIS. Transfacet sacroiliac screw.

\section{Declarations}

\section{Footnotes}

None.

\section{Conflict of interest}

The authors have no actual or potential conflicts of interest to declare.

\section{Authors' contributions}

YP D, QD Y and Y J put forward the concept of this study and designed this experiment, D L, S L and QD Y revised this manuscript. YW W and $\mathrm{Y} \mathrm{J}$ collected data and performed the statistical analysis. All authors read and approved the final manuscript.

\section{Funding}

None.

\section{Ethical approval}

The study was approved by ethics committee of our Hospitals『No. LW2020025 ).

\section{Acknowledgments and Funding Information}

None.

\section{Consent for publication}

The study was undertaken with the patient's written consent.

Availability of data and materials

Not applicable.

\section{References}


1. Salari P, Moed BR, Bledsoe JG. Supplemental S1 fixation for type $C$ pelvic ring injuries: biomechanical study of a long iliosacral versus a transsacral screw. J Orthop Traumatol 2015; 16(4):293-300.

2. Krappinger D, Lindtner RA, Benedikt S. Preoperative planning and safe intraoperative placement of iliosacral screws under fluoroscopic control. Oper Orthop Traumatol 2019; 31(6):465-73.

3. Long T, Li KN, Gao JH, Long TH. Comparative study of percutaneous sacroiliac screw with or without Tirobot assistance for treating pelvic posterior ring fractures. Orthopaedic Surgery 2019; 11(3):38696.

4. Pan ZJ, Hong HX, Huang ZJ,Chen X, Zheng Q, Chen WZ Applied anatomic study of the parameters of screw fixation for the sacroiliac joint. Chinese Journal of Clinical Anatomy 2004; 22(2):125-8.

5. Gras F, Marintschev I, Wilharm A, Klos K, Mückley T, Hofmann GO. 2D-fluoroscopic navigated percutaneous screw fixation of pelvic ring injuries--a case series. BMC Musculoskelet Disord 2010; 11:153.

6. Wei $W$, Wang $H$, Xia ZY, Wang L. The measurement of the screwing trajectory's parameters of the sacroiliac screw for iliosacral joint disruption by the computed tomography. Journal of Hebei Medical University 2018; 39(3):293-6.

7. Bousbaa $\mathrm{H}$, et al. Percutaneous iliosacral screw fixation in unstable pelvic fractures. Pan Afr Med $\mathrm{J}$ $2017 ; 27: 244$.

8. Khaled SA, Soliman OW. Functional outcome of unstable pelvic ring injuries after iliosacral screw fixation: single versus two screw fixation. European Journal of Trauma \& Emergency Surgery 2015; 41(4):387-92.

9. Osterhoff G, Ossendorf C, Wanner GA, Simmen HP, Clément MLW. Percutaneous iliosacral screw fixation in $\mathrm{s} 1$ and $\mathrm{s} 2$ for posterior pelvic ring injuries: technique and perioperative complications. Archives of Orthopaedic and Trauma Surgery 2015; 131(6), 809-13.

10. Grossterlinden L,et al. Factors influencing the accuracy of iliosacral screw placement in trauma patients. Int Orthop 2011; 35(9): 1391-6.

11. Su YG, Wang MY, Wang MY. Intraopertive fluoroscopy in percutaneous sacroiliac screw fixation based on anatomic measurement and digital reconstructed CT data. Chinese Journal of Orthopaedic Trauma 2012; 14(5):405-9.

12. Elzohairy MM, Salama AM. Open reduction internal fixation versus percutaneous iliosacral screw fixation for unstable posterior pelvic ring disruptions.Orthop Traumatol Surg Res 2017; 103(2):223-7.

13. Collinge CA,Crist BD. Combined percutaneous iliosacral screw fixation with sacroplasty using resorbable calcium phosphate cement for osteoporotic pelvic fractures requiring surgery.J Orthop Trauma 2016; 30(6):e217-22.

\section{Tables}

Table 1 Values of anatomical parameters of TFSIS $( \pm s)$ 


\begin{tabular}{|c|c|c|c|c|}
\hline \multirow[t]{4}{*}{$\mathrm{L}(\mathrm{cm})$} & Left & Right & $t \quad P$ & Merged \\
\hline & $10.88 \pm 0.99$ & $10.79 \pm 0.88$ & \multirow{3}{*}{$\begin{array}{l}0.763,0.452 \\
0.178,0,860\end{array}$} & \multirow[t]{3}{*}{$10.84 \pm 0.93$} \\
\hline & Male & Female & & \\
\hline & $10.92 \pm 1.01$ & $10.75 \pm 0.82$ & & \\
\hline \multirow[t]{4}{*}{$\mathrm{M} 1(\mathrm{~mm})$} & Left & Right & \multirow[t]{2}{*}{$1.457,0.981$} & \multirow[t]{4}{*}{$1.14 \pm 0.93$} \\
\hline & $1.08 \pm 0.87$ & $1.20 \pm 0.90$ & & \\
\hline & Male & Female & \multirow[t]{2}{*}{$0.817,0.420$} & \\
\hline & $1.07 \pm 0.83$ & $1.21 \pm 0.91$ & & \\
\hline \multirow[t]{4}{*}{$\mathrm{M} 2(\mathrm{~mm})$} & Left & Right & \multirow[t]{2}{*}{$1.257,0.219$} & \multirow[t]{4}{*}{$5.73 \pm 2.57$} \\
\hline & $5.90 \pm 2.61$ & $5.57 \pm 2.51$ & & \\
\hline & Male & Female & \multirow[t]{2}{*}{$1.762,0,080$} & \\
\hline & $5.61 \pm 2.55$ & $5.85 \pm 2.60$ & & \\
\hline \multirow[t]{4}{*}{$E\left({ }^{\circ}\right)$} & Left & Right & \multirow{4}{*}{$\begin{array}{l}0.523,0.605 \\
0.261,0.796\end{array}$} & \multirow[t]{4}{*}{$53.96 \pm 3.94$} \\
\hline & $53.27 \pm 3.45$ & $54.65 \pm 4.03$ & & \\
\hline & Male & Female & & \\
\hline & $47.46 \pm 5.47$ & $46.67 \pm 4.64$ & & \\
\hline \multirow[t]{4}{*}{$F\left({ }^{\circ}\right)$} & Left & Right & \multirow[t]{4}{*}{$1.361,0.184$} & \multirow[t]{4}{*}{$47.47 \pm 5.13$} \\
\hline & $47.13 \pm 5.26$ & $47.81 \pm 5.01$ & & \\
\hline & Male & Female & & \\
\hline & $48.46 \pm 4.80$ & $46.47 \pm 4.75$ & & \\
\hline \multirow[t]{4}{*}{$A\left({ }^{\circ}\right)$} & Left & Right & \multirow[t]{4}{*}{$1.188,0.245$} & \multirow[t]{4}{*}{$13.91 \pm 2.92$} \\
\hline & $14.11 \pm 2.91$ & $13.70 \pm 2.99$ & & \\
\hline & Male & Female & & \\
\hline & $14.26 \pm 3.00$ & $13.56 \pm 2.97$ & & \\
\hline \multirow[t]{4}{*}{$\mathrm{B}\left({ }^{\circ}\right)$} & Left & Right & \multirow[t]{4}{*}{$1.008,0.276$} & \multirow[t]{4}{*}{$8.57 \pm 1.63$} \\
\hline & $8.67 \pm 1.63$ & $8.49 \pm 1.56$ & & \\
\hline & Male & Female & & \\
\hline & $8.78 \pm 1.64$ & $8.36 \pm 1.50$ & & \\
\hline $\mathrm{D} 1(\mathrm{~mm})$ & Left & Right & $1.232,0.252$ & $11.91 \pm 1.47$ \\
\hline
\end{tabular}




\begin{tabular}{|lllll|} 
& $11.93 \pm 1.46$ & $11.90 \pm 1.48$ & & \\
& Male & Female & $1.378,0.201$ & \\
\cline { 2 - 3 } D2(mm) & Left & Right & $0.671,0.504$ & $7.75 \pm 0.89$ \\
\cline { 2 - 3 } & $7.77 \pm 0.90$ & $7.72 \pm 0.88$ & $1.451,0.141$ & \\
& Male & Female & & \\
\cline { 1 - 3 } & $7.87 \pm 0.92$ & $7.62 \pm 0.85$ & \\
\hline
\end{tabular}

\section{Figures}
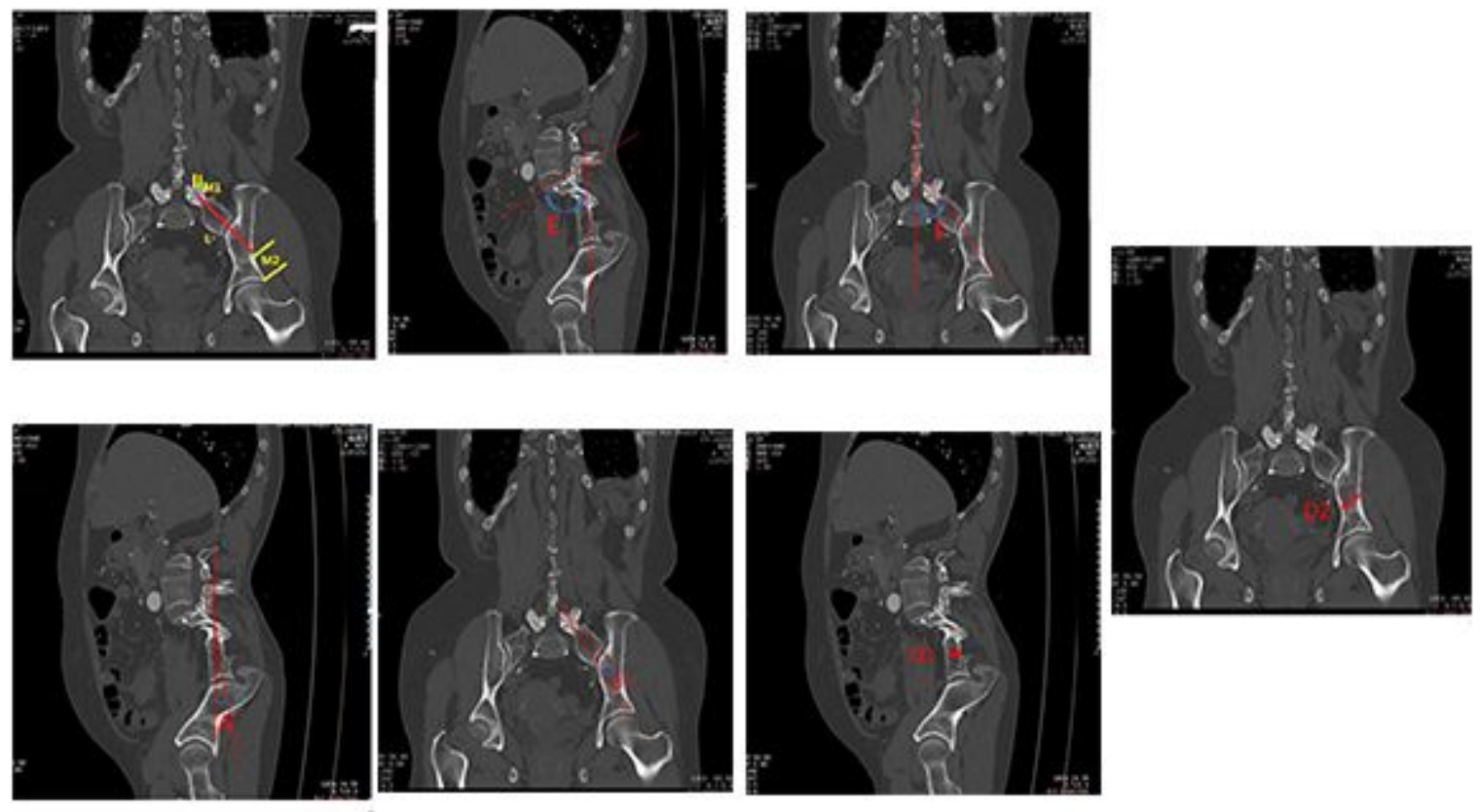

Figure 1

Definitions of anatomical parameters a:the length of screw pathway (L), the insertion point position (M1) and the exit point position (M2) b: the anteversion angle (E) c: the outward angle (F) d: the forward safety angle (A) e: the outward safety angle (B) f: the height (D1) g: the width (D2) 

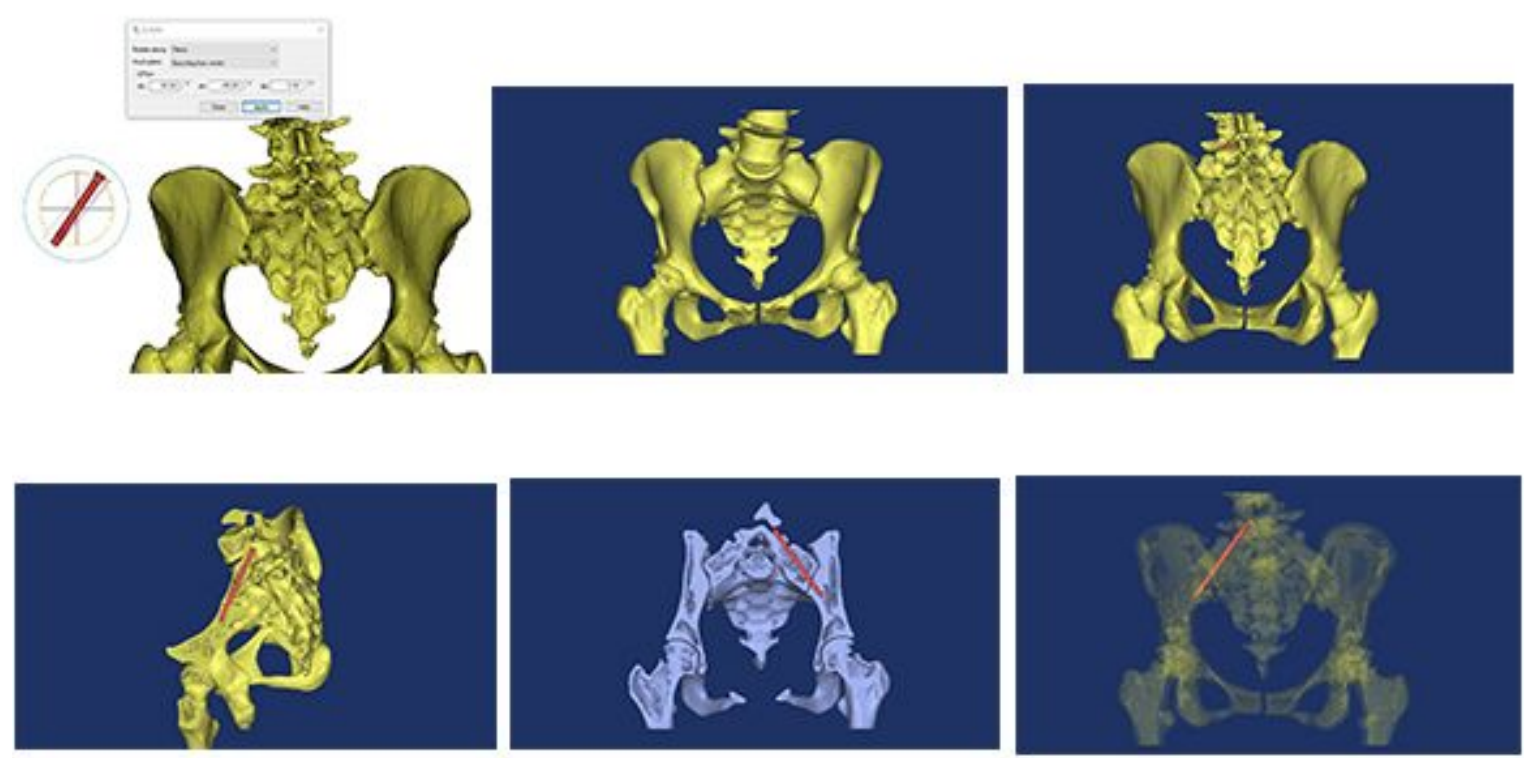

Figure 2

Simulated placement of TFSIS a: 3-D reconstructions of pelvis and $5 \mathrm{~mm}$ diameter screw b: anterior view after simulated placement c: posterior view after simulated placement d: sagittal view after simulated placement e: coronal view after simulated placement f: perspective view after simulated placement 